Annuaire suisse de politique de développement

8 | 1988

Annuaire Suisse - Tiers Monde 1989

\title{
Regards sur l'Asie : une formidable redistribution des cartes économiques
}

\section{Gilbert Etienne}

\section{(2) OpenEdition}

1 Journals

Édition électronique

URL : http://journals.openedition.org/aspd/1280

DOI : $10.4000 /$ aspd. 1280

ISSN : 1663-9669

Éditeur

Institut de hautes études internationales et du développement

\section{Édition imprimée}

Date de publication : 1 décembre 1988

Pagination : 167-177

ISSN : 1660-5934

\section{Référence électronique}

Gilbert Etienne, «Regards sur l'Asie : une formidable redistribution des cartes économiques », Annuaire suisse de politique de développement [En ligne], 8| 1988, mis en ligne le 13 mars 2013, consulté le 08 septembre 2020. URL : http://journals.openedition.org/aspd/1280 ; DOI : https://doi.org/10.4000/ aspd. 1280 


\title{
Regards sur l'Asie : une formidable redistribution des cartes économiques
}

\author{
Gilbert Etienne
}

Le Tiers Monde va mal dit-on volontiers. Dans les deux tiers des pays qui le composent les niveaux de vie reculent depuis 1980 ou parfois plus tôt: Cela touche la santé, l'éducation, l'alimentation, l'emploi... Si au lieu de compter les Etats, on considère les populations, le tableau change de couleur. L'Asie qui, Moyen-Orient non compris, abrite deux ressortissants sur trois du Tiers Monde, affiche un bulletin de santé plutôt favorable. La plupart des pays connaissent un taux de croissance relativement élevé qui s'accompagne d'un progrès social non négligeable. Nombre de pays ont même amélioré leurs performances depuis 1980 .

Fait encore plus significatif, les changements économiques dont ce continent est le théâtre sont en train de prendre des dimensions planétaires. Ils constituent l'amorce d'une redistribution des cartes financières, économiques, même technologiques au niveau de l'ensemble du globe.

\section{La rentrée en scène}

1905, les étudiants de Bombay et de Calcutta allument les lampions, ceux de I'Indochine française et des Indes néerlandaises font de même. Après cent cinquante ans de conquêtes, de domination lourde d'arrogance, pour la première fois une grande puissance européenne, la Russie (certes vermoulue) est écrasée par des Asiatiques: les croiseurs japonais mettent en pièce sa flotte, sur terre l'artillerie et l'infanterie nipponnes anéantissent les divisions du tsar. Le Japon, «nouveau pays industriel» avant la lettre, confirme par les armes sa maîtrise des techniques occidentales. II est en train de sortir de ce que l'on appelera plus tard le sous-développement. 
Depuis lors, le reste de l'Asie s'affirme. De 1945 à 1950, la plupart des pays retrouvent leur indépendance et prennent en main leur développement. Aujourd'hui, en cette fin du XXe siècle, c'est presque tout le continent qui bouillonne d'activité économique avec, dans la plupart des cas, le rejet des aventures militaires. En 1960, Amérique du nord et Europe occidentale assuraient $58 \%$ de la production mondiale contre $11 \%$ pour l'Asie (Moyen-Orient non compris), pourcentages qui passent respectivement à 51 et 19 en 1981. En l'an 2000, la part de l'Asie risque de dépasser celle de l'Amérique du nord $(21,4 \%)$ et celle de l'Europe occidentale (23,3\%). A lui seul, l'Extrême-Orient devrait atteindre $19 \%$. Ces estimations japonaises ont beau être grossières, elles indiquent bien les tendances.

\section{Victoire sur la famine}

Grâce à des politiques agricoles dans l'ensemble judicieuses (progrès technique, prix, constitution de réserves de grains), la plupart des pays d'Asie ont connu une hausse sensible de leur production agricole au point que les importations de céréales reculent, parfois disparaissent. Les pays les plus riches comme la Corée du Sud importent des céréales non pour nourrir leur population mais pour alimenter le bétail, comme le font les Japonais et les Européens. Pour la première fois dans l'histoire, l'Asie est à peu près à l'abri d'effroyables famines. En 1987, malgré des conditions météorologiques désastreuses dans de nombreux pays, ceux-ci sont parvenus à éviter une rupture du front alimentaire grâce à leurs stocks et aussi à des mesures de secours qui, même dans le fragile Bangladesh, ont fonctionné de manière assez satisfaisante.

Cette alerte qui va, au moins temporairement, relancer les importations de grain, nous montre que la partie n'est pas encore entièrement gagnée comme le mesurent fort bien d'ailleurs les gouvernements. II reste capital de continuer à promouvoir l'essor de l'agriculture dont le poids reste lourd sur l'ensemble de l'économie. De plus, la sécurité alimentaire d'aujourd'hui ne veut pas dire qu'il n'existe plus de gens mal nourris, trop pauvres pour acheter davantage de grain. En Inde, au Bangladesh, dans une moindre mesure en Chine, la misère sous ses formes les plus aiguës n'a pas disparu jusqu'à maintenant. A ces quelques réserves, la consolidation de l'agriculture représente une gros atout.

\section{A l'intérieur de l'Asie}

D'autres mutations non moins profondes se déroulent au sein de l'Asie. Pendant deux mille ans, jusqu'à la fin du XVIIle siècle, I'Inde a joué un rôle prépondérant dans les échanges internationaux en direction du Moyen-Orient et de l'Europe, vers l'Asie du Sud-Est et l'Extrême-Orient. Ses exportations sont largement excédentaires. Pline l'Ancien se plaint que l'or de Rome serve à payer épices, cotonnades et soieries indiennes. Plus tard, au temps de Louis XIV, Bernier explique à Colbert comment l'or et l'argent du monde entier, y compris les métaux précieux des Amériques, finissent en Inde. 
Contraste étonnant aujourd'hui. Le Japon a pris la tête du continent, mais d'autres le suivent. Après avoir joué pendant de longs siècles un rôle effacé, la petite île de Taïwan, repaire de pirates au XVIle siècle, vient derrière le Japon au deuxième rang mondial des réserves en devises, avec 75 milliards de dollars. Jusqu'en 1876, la Corée était restée si fermée au monde extérieur que, non sans agacement, les chancelleries occidentales l'avaient baptisée le "royaume ermite». II y a quatre ans, les Sud-Coréens n'exportaient pratiquement pas d'automobiles. En 1987, ils en déversent 700.000 sur le marché mondial. Leurs aciéries sont en train de devenir les premières du monde. Leurs biens de consommation voyagent partout et leurs industriels vont jusqu'en Afrique noire monter des usines textiles! Quant à ces têtes d'épingles sur la carte que sont Hong Kong et Singapour, elles ne cessent de s'affirmer comme centres industriels et bancaires.

Les pays de l'Asie du Sud-Est n'affichent pas des performances aussi spectaculaires. Les Philippines restent empêtrées dans leurs dettes et leurs embarras politiques mais l'économie se reprend. L'Indonésie, qui a plus intelligemment utilisé ses revenus pétroliers que le Mexique et le Nigéria, passe par une phase délicate à la suite de la baisse des cours de l'or noir. Les Thaïs, avec leur air décontracté, commencent à talonner l'Extrême-Orient. Leur économie se diversifie, les exportations montent, surtout par le biais de petites et moyennes entreprises. Derrière le riz et le maïs viennent les textiles, l'agro-alimentaire, l'électronique... La Thaïlande s'assure aujourd'hui $35 \%$ du marché mondial de l'ananas en boîte.

Reste I'Asie du Sud, Inde, Pakistan, Bangladesh. Ce dernier constitue un des maillons les plus faibles du continent, à la suite d'un destin cruel depuis la fin du XVIIle siècle, de calamités naturelles dramatiques, d'une pression démographique accablante, de facteurs politiques...

De son côté, l'Inde paye cher la politique de développement trop peu ouverte sur le monde extérieur qu'elle a suivie dans les décennies 1960 et 1970. Les heureux correctifs apportés par les réformes en cours commencent à porter leurs fruits. Toutefois, ce pays qui était la dixième puissance industrielle dans le monde en 1950, peu après son indépendance, est tombé au vingtième rang. Quant à sa part du commerce mondial, elle s'est beaucoup rétrécie.

Dans un contexte politique différent, la Chine a suivi sous Mao Zedong une politique d'industrialisation qui s'apparente à celle de l'Inde. Elle aussi joue, non sans brio, la carte de l'ouverture tous azimuts. Le commerce extérieur longtemps languissant s'est beaucoup développé. Néanmoins les exportations restent assez loin derrière celles de la petite île de Taiwan!

\section{Les réformes économiques}

Ce climat dynamique est étroitement lié aux politiques judicieuses de développement suivies de longue date par le Japon, et, dès les années 1960, par la Corée du Sud et l'île de Taiwan. Ces deux pays, pas plus d'ailleurs que le Japon, n'incarnent une sorte de libéralisme classique et de laisser-faire. Au con- 
traire, l'Etat intervient, de manière prononcée, dans la marche de l'économie, mais il le fait à bon escient. En Corée, à Taiwan, le secteur public tourne de manière efficace. Les entreprises privées sont soutenues, voire stimulées par les autorités.

Dans beaucoup d'autres pays d'Asie, les politiques plus ou moins socialistes, teintées de populisme au gré des circonstances, n'ont pas donné les résultats escomptés, ni en terme de croissance, ni sur le plan de justice sociale. Depuis grosso modo 1975-80, s'est levé un vent de réformes pratiquement partout, au point qu'il commence même à souffler aujourd'hui sur le Viet Nam et la Birmanie. De Pékin à Islamabad, en passant par l'Asie du Sud-Est, le dogmatisme et la rhétorique reculent devant une ligne beaucoup plus pragmatique. II importe de rendre les secteurs publics de l'industrie plus performants, de libéraliser le secteur privé, d'alléger les filières bureaucratiques, ce qui en même temps réduit la corruption. II est non moins impératif d'adopter une politique économique plus ouverte sur le monde extérieur. Transferts de technologie, investissements privés étrangers sont beaucoup plus vivement encouragés que par le passé.

En substance tous ces pays aspirent à produire plus, mieux et à meilleur coût afin de répondre au besoin de leurs marchés intérieurs et de pousser leurs exportations de biens manufacturés. Pour ce faire des pays comme la Chine, l'Inde, le Pakistan, doivent moderniser leurs équipements industriels souvent vétustes et créer de nouvelles usines.

La partie est encore loin d'être gagnée et les réformes doivent aller plus loin. Cependant les premiers résultats sont encourageants. L'économie chinoise a reçu un gros coup de fouet depuis la mort de Mao Zedong. Celle de l'Inde améliore sa cadence depuis 1980, comme celle du Pakistan.

\section{Adieu aux critères du sous-développement}

Cette Asie à plusieurs vitesses bouscule les critères classiques du sous-développement et du dialogue nord-sud. Le vieux pacte colonial selon lequel les pays occidentaux livraient des biens manufacturés contre des matières premières est mort et enterré. Biens semi-durables, articles de consommation courante, équipements circulent dans les deux sens.

Les multinationales occidentales ne sont plus seules sur la scène. Les entreprises japonaises, industries, banques, étendent leurs tentacules. De Hong Kong et Singapour se tissent d'autres toiles d'araignée couvrant l'Asie du Sud-Est. La République populaire de Chine investit dans des mines de fer en Australie et dans les forêts canadiennes. Les chaînes d'hôtels indiens s'allongent jusqu'à New York et Moscou, tandis que des industriels indiens créent des fabriques en Afrique. Les entreprises de construction indiennes et pakistanaises sont actives au Moyen-Orient, en Libye, en Algérie.

Le Japon qui, pendant longtemps s'était concentré sur l'Extrême-Orient et l'Asie du Sud-Est, s'affirme de plus en plus sur les marchés de l'Inde, du Pakistan, du Bangladesh. La Corée lui emboîte le pas. 
La fluidité des relations économiques est aussi liée aux salaires dans une sorte de jeu de dominos à l'envers. Les firmes étrangères en quête de main d'oeuvre bon marché ne vont plus à Hong Kong. Iront-elles encore longtemps à Taiwan et en Corée du Sud où les salaires montent? Dès lors, les investisseurs japonais vont aux Philippines et les Coréens au Bangladesh!

Lorsqu'on jette un coup d'oeil au commerce extérieur du continent, on peut se demander si ceux qu'on dénomme les "quatre petits dragons" sont vraiment petits (Hong Kong, Singapour, Corée du Sud, île de Taiwan) et si les grands, Chine, Inde, sont vraiment grands! Sous cet angle encore, vieux schémas et idées reçues sont rudement ébranlés.

\section{Exportations et importations par pays}

\begin{tabular}{|c|c|c|}
\hline & $\begin{array}{l}\text { Exportations } \\
\text { (milliards de \$) }\end{array}$ & Importations \\
\hline \multicolumn{3}{|l|}{ Année 1987} \\
\hline $\begin{array}{l}\text { Japon } \\
\text { lle de Taiwan } \\
\text { Corée du Sud } \\
\text { Chine } \\
\text { Inde }\end{array}$ & $\begin{array}{l}229.0 \\
54.0 \\
45.0 \\
38.2 \\
13 \text { à } 15\end{array}$ & $\begin{array}{l}149.0 \\
35.0 \\
40.5 \\
41.0 \\
18.0\end{array}$ \\
\hline \multicolumn{3}{|l|}{ Année 1986} \\
\hline $\begin{array}{l}\text { Hong Kong } \\
\text { Singapour } \\
\text { Indonésie } \\
\text { Thailande }\end{array}$ & $\begin{array}{l}35.4 \\
22.4 \\
14.8 \\
8.7\end{array}$ & $\begin{array}{c}35.3 \\
25.5 \\
10.7 \\
9.2\end{array}$ \\
\hline
\end{tabular}

Entre 1960 et 1970, les exportations chinoises et indiennes (un peu plus de 2 milliards de dollars) dépassaient celles de la Corée du Sud et de Taiwan.

\section{Des explications incertaines}

Des hommes d'affaires, des universitaires, américains et européens, expliquent les succès du Nouveau monde sinisé, chinois ou marqué par la civilisation chinoise, en évoquant le confucianisme, son sens de la discipline, de la cohésion, du labeur... En va-t-il bien ainsi? Au début de siècle, Occidentaux et Chinois progressistes attribuaient le déclin de la Chine au dit-confucianisme! Aujourd'hui, la Chine proprement dite - le handicap de sa lourde masse mis à part affiche des performances moins spectaculaires que celles de ses voisins. En affinant l'analyse, on repère des différences assez sensibles entre les conditions de développement dans l'île de Taiwan et en Corée du Sud.

*Titre du Livre de L.Vandermeersch, Paris, PUF, 1986. 
Que disent les intéressés? Prenons l'absence relative de grèves au Japon, attribuée aux vertus confucéennes. Lors d'un colloque à l'INSEAD (Fontainebleau) un économiste japonais rappelait ceci: jusque vers 1950, les conflits sociaux avaient été très durs. Après cette date, patrons et ouvriers ont compris la stérilité de ces luttes et l'intérêt réciproque à trouver, à temps, des accommodements. En somme, une victoire du bon sens comparable à celle que nous avons remportée en Suisse, il y a un demi-siècle... sans être confucéens!

Autre exemple, l'opiniâtreté au travail. Ceux qui visitent les usines du groupe Tata en Inde trouvent un haut degré d'efficacité, une productivité élevée. Même dans le si pauvre Bangladesh, des fabriques de vêtements tournent - et vite pour l'exportation. Déjà vers 1960, dans les usines de Péchiney au Cameroun et en Guinée, la main-d'oeuvre africaine bien sélectionnée, bien encadrée et payée décemment, fournissait des prestations comparables à celles de beaucoup d'ouvriers français.

Par ces exemples nous ne prétendons pas enlever toute signification aux facteurs culturels de développement. C'est vrai que ceux-ci peuvent exercer des effets positifs ou négatifs, mais ils ne sont pas immuables et on ne doit pas trop en grossir l'importance.

Faut-il alors évoquer les facteurs politiques? Réponse à nouveau malaisée. La forte croissance du Japon s'est accommodée aussi bien d'un pouvoir totalitaire dans l'entre-deux-guerres que d'un système démocratique après 1945. Hong Kong vit sous un régime colonial, certes de bon aloi! Les régimes musclés de Corée du Sud et de Taiwan sont en pleine évolution tandis que dans bien d'autres pays du Tiers Monde, les dictateurs ont enregistré de piètres scores économiques.

Souvent le succès tient au choix de la politique de développement, à des efforts d'imagination, aux interventions judicieuses de l'Etat, à la bonne décision prise au bon moment. En misant dès le début des années 1960 sur les industries d'exportation, Corée du Sud et Taiwan ont profité de l'âge d'or du commerce international. Les Indiens, eux, ont pris le "bus" tard, entre 1970 et 1980, sinon après. D'innombrables autres faits pourraient être avancés, par exemple la gestion des stocks «just in time» lancée par les firmes japonaises ou ces firmes automobiles dont les ouvriers terminent les voitures (peinture) durant le trajet en mer, de l'archipel nippon aux ports américains.

Autre trait, celui-ci majeur, c'est la fantastique capacité d'adaptation aux changements de conjoncture, la capacité à retomber sur leurs pieds et rebondir que montrent les économies du Japon et des "quatres dragons".

En fin de compte rappelons le poids de l'histoire, le degré de développement économique et technique atteint par les civilisations d'Asie déjà avant l'ère coloniale. A cet égard le continent dispose d'atouts qui manquent à l'Afrique au sud du Sahara et, même, à l'Amérique latine.

Rien n'est jamais définitivement joué et d'aucuns prédisent aux Coréens et autres des jours difficiles, devant la montée du protectionnisme et la concurrence accrue entre pays d'Asie, notamment la poussée des exportations de Chine et d'Inde dans les produits manufacturés. 
Malgré d'éventuels aléas, force est de constater la rentrée en scène de ces vieilles civilisations, en partie rajeunies, en partie fidèles à leurs héritages culturels: mouvement spectaculaire du peloton de tête, mouvement qui s'accélère en Chine, en Asie du Sud-Est, en Inde, au Pakistan.

\section{Et nous autres Occidentaux?}

Les Américains participent activement par leurs investissements, leurs transferts de technologie, leurs relations commerciales au grand jeu asiatique. Leur côte ouest complète ce puissant ensemble économique centré sur l'océan Pacifique. Le commerce des Etats-Unis avec l'Asie dépasse aujourd'hui celui qu'ils entretiennent avec l'Europe occidentale.

Cette dernière paraît plutôt timide. Certes, les investissements de la RFA, du Royaume Uni, des Pays-Bas, de la Suisse sont en hausse. Les Français et les Italiens commencent à se faire remarquer. Nos banques se sont implantées un peu partout. Mais voyons-nous assez loin et assez grand? Lors d'un récent colloque du Centre Asie de l'Institut universitaire des hautes études internationales, un financier suisse revenant de l'Extrême-Orient s'exclamait: "Là-bas, ça bouge dans tous les sens, chez nous, à Berne ou à Bruxelles, on discute depuis trente ans du prix des pommes de terre".

Les pays d'Asie cherchent à diversifier leurs relations au-delà du Japon et des Etats Unis. Nous aurions intérêt à mieux répondre à leur attente. Sinon, ils pourraient très bien se passer de nous. Ils trouveront tout ce dont ils ont besoin chez les Japonais et les Américains, d'autant plus que ces marchés sont largement ouverts à leurs produits.

II faut se demander si nos élites dirigeantes et nos opinions publiques sont assez conscientes de ces enjeux. Faisons-nous assez d'efforts pour connaître ces pays? On sait le soin que mettent les Japonais à étudier leurs clients présents et futurs. Formons-nous assez de spécialistes dans nos universités d'Europe? Même si les temps de travail vont peu à peu baisser en Extrême-Orient, les pays européens ont-ils intérêt à augmenter la durée des vacances, réduire les horaires de travail, abaisser l'âge de la retraite?

\section{L'argument de l'Europe 1993}

A ces arguments, des Européens d'esprit ouvert répondent que la nouvelle communauté qui débutera en 1993 va offrir d'énormes débouchés à l'intérieur de ses frontières: l'Espagne - le mouvement est déjà en marche - le Portugal, la Grèce... Alors pourquoi chercher midi à quatorze heures en Asie, sur des marchés lointains, difficiles? C'est tout de même plus simple d'ouvrir un bureau à Lisbonne qu'à Pékin ou Bombay.

Dans quelle mesure cet argument est-il pertinent ? Les avis recueillis jusqu'à maintenant sont partagés. Certains l'acceptent, d'autres le rejettent en faisant 
remarquer que le potentiel à l'intérieur de l'Europe pourrait ne pas correspondre aux espoirs.

Une question capitale qui mériterait - et sans tarder - des études approfondies !

\section{Et la Suisse ?}

Comment apparaît la Suisse dans cette perspective d'une Asie en plein essor et de l'Europe de 1993 ? Etant donné que nous nous bornerons à passer des accords d'association avec la CEE sans y adhérer, nous avons plus que nos voisins intérêt à garder deux fers au feu : les marchés proches et ceux d'Asie.

La présence commerciale suisse dans cette partie du monde est ancienne. Elle se renforce et s'organise au XIXe siècle grâce à de nombreuses maisons, nos Welthandelsfirmen qui ouvrent des comptoirs au Japon, à Bombay, à Singapour, Bangkok, Colombo, Shanghaï, Hong Kong... Concentrées sur l'export-import, ces entreprises, du moins un certain nombre d'entre elles, s'élargissent après la décolonisation. Elles créent à côté de leurs imports-exports des usines, tandis que nos multinationales (chimiques, alimentaires, machines) accroissent leurs activités commerciales et investissent dans des fabriques sur place.

Ce mouvement déjà très net dans les années 1950 à 60 (à cette époque la Suisse vient au troisième rang des investissements privés étrangers en Inde) se poursuit aujourd'hui. Néanmoins, la concurrence augmente : aux Occidentaux s'ajoutent les Japonais suivis des Coréens et bientôt des Chinois de Taïwan.

Faute de statistiques assez détaillées, il est malaisé de préciser les courants d'investissement privées suisses en Asie, en dehors du Moyen-Orient. Le Japon occupe, de loin, la première place avec environ deux milliards de francs à la fin de l'année 1987. Il est suivi par Hong Kong (plus de 200 millions de francs). On tombe ensuite à 140-100 millions à Singapour, en Malaisie, en Inde et en dessous de 100 millions pour les autres pays. Seul point clair - et assez troublant - , la place relativement limitée des investissements destinés à l'Asie.

En ce qui concerne nos banques, leur intérêt pour l'Asie (Japon, Hong Kong, Singapour exceptés) est beaucoup plus tardif. En 1958, un très haut fonctionnaire indien venait sonder les milieux financiers suisses; il était reçu dans une parfaite indifférence !

Autour de 1980, voire un peu avant, nos banquiers ont commencé à changer de cap. Missions exploratoires, contacts multiples, ouvertures de bureaux de représentation et d'agences dans de nombreux pays, notamment la Chine, l'Inde, l'Asie du Sud-Est. Même nos banques privées commencent à s'aventurer sur ces marchés.

Ce renforcement de la présence suisse en Asie est complété par les différentes formes de coopération qui prennent corps dans les années 1960: assistance technique, crédits mixtes, activités de nombreuses ONG suisses. 


\begin{tabular}{|c|c|c|c|c|}
\hline \multicolumn{5}{|c|}{ Vue d'ensemble des crédits mixtes à l'Asie (1987) } \\
\hline & & \multicolumn{3}{|c|}{ (montant en millions de frs) } \\
\hline & & Confédération & Banques & Total \\
\hline \multirow{2}{*}{$\begin{array}{l}\text { SRI LANKA } \\
\text { rallonge }\end{array}$} & $(05.11 .79)$ & 15.0 & 15.0 & $30.0^{*}$ \\
\hline & (27.11.87) & 0.7 & 0.7 & $1.4^{*}$ \\
\hline THAILANDE I & $(01.06 .79)$ & 12.7 & 38.3 & $51.0^{*}$ \\
\hline INDE & (23.04.84) & 40.0 & 60.0 & 100.0 \\
\hline THAILANDE ॥ & (24.01.85) & 20.0 & 40.0 & $60.0^{*}$ \\
\hline rallonge & $(17.12 .87)$ & 1.5 & 3.0 & $4.5^{\star}$ \\
\hline JORDANIE & (19.02.86) & 20.0 & 40.0 & 60.0 \\
\hline INDONESIE & (20.03.86) & 51.0 & 102.0 & $153.0^{*}$ \\
\hline CHINE || 1) & (09.12.87) & 40.0 & 60.0 & 100.0 \\
\hline PAKISTAN ${ }^{1)}$ & $(07.12 .87)$ & 31.5 & 58.5 & 90.0 \\
\hline \multicolumn{5}{|c|}{ Crédits mixtes en préparation } \\
\hline & & Confédération & Banques & Total \\
\hline \multirow{2}{*}{\multicolumn{2}{|c|}{$\begin{array}{l}\text { THAILANDE III } \\
\text { PHILIPPINES I }\end{array}$}} & 28.0 & 52.0 & 80.0 \\
\hline & & 31.5 & 58.5 & 90.0 \\
\hline
\end{tabular}

1) part de la Confédération = DON

* montant totalement engagé

La part de l'Asie dans le budget de la DDA a eu tendance à baisser au fil des années, en faveur de l'Afrique, ce qui est logique compte tenu des besoins de ce continent. C'est ainsi que sur un montant de 363 millions de francs de la coopération technique et aide financière bilatérale de la Confédération (sans l'aide humanitaire et les crédits mixtes) l'Asie reçoit 87 millions, soit $24,2 \%$ (année 1987).

La place de la région dans notre commerce extérieur a évolué comme suit:

\section{Evolution et structure du commerce extérieur suisse.}

A

ASIE (sans Moyen-Orient)

Moyen-Orient*

Europe

Afrique

Amérique du Nord

Amérique centrale/Amérique du Sud
1980

Importations Exportations

(en milliards de \$)

3.67

3.71

1.5

3.23

48.31

33.32

1.56

2.41

4.43

3.97

1.28

2.29

Totaux

60.86

49.60 
B

1987

Importations Exportations

(en milliards de \$)

ASIE (sans Moyen-Orient)

5.97

7.29

Moyen-Orient ${ }^{\star}$

0.69

3.16

Europe

61.05

45.94

Afrique

1.39

1.67

Amérique du Nord

4.29

6.55

Amérique centrale/Amérique du Sud

1.69

2.15

Totaux

75.17

67.48

* Moyen-Orient : pays arabes d'Asie, Israël, Iran

** Le total ne correspond pas entièrement aux chiffres par région. Ceux-ci ont été arrondis, les chiffres pour l'Océanie ne sont pas compris dans le tableau.

Il est intéressant de voir la répartition en pour cent de notre commerce extérieur. Face à la stabilité de nos exportations vers l'Europe, la hausse de nos marchés asiatiques est significative.

\section{Répartition des exportations en \%}

1980

1987

ASIE (sans Moyen-Orient)

7.5

10.8

Moyen-Orient

7.0

4.7

Europe

67.1

68.1

Afrique

4.8

2.5

Amérique du Nord

8.0

9.7

Amérique centrale et du Sud

4.6

3.2

Cette évolution reflète assez bien l'évolution de l'économie mondiale. La fin du boom du pétrole affecte nos ventes au Moyen-Orient et en Afrique du Nord. II en va de même des graves difficultés de l'Afrique noire et de l'Amérique latine. Les taux de l'Europe et de l'Amérique du Nord montent quelque peu, tandis que c'est en Asie que nos exportations progressent le plus. Vient en tête le Japon (2,6 milliards de francs d'exportations en 1987) suivi par Hong Kong (1,4 milliard), la Chine (614 millions), Taïwan et Singapour (près de 500 millions chacun), l'Inde (354 millions) la Corée du Sud (328 millions). Inversement, il faut noter que la Suisse, malgré sa politique commerciale très libérale, subit sans 
trop de mal le choc des produits de Taïwan et de Corée. Notre balance commerciale est positive face à la première et très légèrement négative face à la seconde (-27 millions de francs).

En conclusion, la Suisse dispose d'atouts favorables. Elle est implantée de longue date dans le continent. Sa collaboration est recherchée et appréciée. Mais, sommes-nous assez actifs? En ce qui concerne le Japon et Hong Kong, la réponse est plutôt positive. A propos des autres pays nous sommes moins sûrs. 